\title{
Robustness of the ATLAS pixel clustering neural network algorithm
}

\section{Edvin Sidebo On behalf of the ATLAS Collaboration*}

Physics Department, Royal Institute of Technology, Stockholm, Sweden

E-mail: edvin.sideboecern.ch

Proton-proton collisions at the energy frontier puts strong constraints on track reconstruction algorithms. In particle-dense environments multiple charged particles can contribute to the same cluster of neighbouring read-out elements in the ATLAS pixel detector. The ATLAS track reconstruction algorithm utilises an artificial neural network to identify and split such clusters. The robustness of the neural network algorithm is presented, probing its sensitivity to uncertainties in the detector conditions. The robustness is studied by evaluating the stability of the algorithm's performance under a range of variations in the inputs to the neural networks. Within reasonable variation magnitudes, the neural networks prove to be robust to most variation types.

Fourth Annual Large Hadron Collider Physics

13-18 June 2016

Lund, Sweden

${ }^{*}$ Speaker. 


\section{Introduction}

The reconstruction of charged particles requires precise measurements of the position of the particles as they traverse the detector. In particle-dense environments multiple charged particles can be separated by distances comparable to the size of the read-out elements of the detectors. This would lead to multiple charged particles contributing to a single cluster of neighbouring read-out elements. Hence, in these environments it is particularly challenging to determine the position of charged particles in the detector. The ATLAS track reconstruction algorithm [1] utilises artificial neural networks (NNs) to identify pixel clusters created by multiple charged particles in the ATLAS pixel detector and to estimate the positions of the particles. This study evaluates the robustness of these NNs. The NNs are trained with Monte Carlo (MC) simulated samples to classify clusters according to the number of particles that created them, and to estimate the particle positions. Uncertainties in the modelling of the detector response and the charge calibration of the pixel detector can lead to suboptimal training of the NNs and subsequent differences in the tracking performance between the recorded data and the simulation. The robustness of the NNs is probed by measuring their response to a set of variations to their input. What is presented here is a summary of the study presented in Ref. [2].

The pixel detector is a high-granularity silicon detector, segmented in $R \phi$ and $z$ and is the innermost part of ATLAS [3]. The study is limited to the barrel of the pixel detector, which consists of four layers where the innermost layer (Insertable B-layer, IBL) [4] was installed between run 1 and run 2 of the LHC. The charge deposited in each pixel is a key quantity for determining whether a cluster has been created by multiple particles. The charge in a pixel is read out as time-over-threshold. In the pixel detector local coordinate frame, the local $x$ and $y$ directions approximately coincide with the global $R \phi$ and $z$ directions, respectively. ${ }^{1}$ In the innermost layer (IBL), most pixels have dimensions $50 \times 250 \mu \mathrm{m}$ in the local $x \times$ local $y$ directions, whereas in the other layers of the pixel detector most have dimensions $50 \times 400 \mu \mathrm{m}$.

In the track reconstruction algorithm, candidate tracks are formed from measurements in the inner detector.Tracks which share measurements typically have a high probability to be discarded, to suppress the number of duplicated or fake tracks. However, when multiple particles contribute to the same pixel cluster, multiple tracks will naturally share this cluster. The NNs are used to recover such tracks that would otherwise be lost [5].

\section{Pixel Neural Networks}

The two sets of NNs used in the ATLAS track reconstruction are the "particle multiplicity NN", used to determine the number of particles associated with a pixel cluster, and the "position NN", used to assign the positions of those particles. Each is a multi-layer perceptron with two hidden

\footnotetext{
${ }^{1}$ ATLAS uses a right-handed coordinate system with its origin at the nominal interaction point (IP) in the centre of the detector and the $z$-axis along the beam pipe. The $x$-axis points from the IP to the centre of the LHC ring, and the $y$-axis points upwards. Cylindrical coordinates $(R, \phi)$ are used in the transverse plane, $\phi$ being the azimuthal angle around the $z$-axis.
} 
layers. For a detailed description of the NNs, their implementation and performance in data see Refs. [6, 7]. The particle multiplicity and position NNs share a common set of 60 input quantities, characterising the properties of pixel clusters. The most important ones are the individual pixel charges and the local track-to-module incidence angles.

The particle multiplicity NN is trained on simulated events with the number of particles traversing the pixel cluster as the target value. Its output corresponds to the probability that a cluster is associated with one, two or three or more particles. The position NNs are trained with positions of the intersections of the particle trajectories with the pixel module with respect to the cluster centroid as target values. Their output is a set of one, two or three pairs of local $x$ and $y$ positions, depending on the particle multiplicity. The NNs are validated using a statistically independent sample by comparing their response to the target values on which they have been trained. The performance of the particle multiplicity $\mathrm{NN}$ is evaluated using the metrics single-particle fake rate, the fraction of single-particle clusters that are wrongly identified as multi-particle; and multi-particle efficiency, the fraction of multi-particle clusters that are correctly identified as such. The performance of the position $\mathrm{NN}$ is evaluated by examining the means and widths of the residual distributions, the latter calculated as the differences between the position NN estimates and the target values.

\section{Results}

Five different types of variations are applied to the input, manipulating the cluster charge and the track incidence angles. Cluster charge variations probe uncertainties in the measured charge, originating from charge calibration and simulation, which could potentially bias the training of the NNs. Overall, the NNs are found to be robust against reasonably sized variations. The one variation which has the largest impact on the particle multiplicity $\mathrm{NN}$ is the one meant to emulate the uncertainty on the charge calibration scale. It is performed by multiplying each pixel's charge by a factor equal to $0.7,0.8,0.9,1$ (default), 1.1, 1.2 and 1.3. The charge calibration scale is expected to be accurate to $10 \%$. Pixels which after the variation have a charge below threshold are still kept so as to preserve the original cluster shape. In Fig. 1 the single-particle fake rate and multi-particle efficiency are shown as a function of a constant pixel charge scale factor. Both the single-particle fake rate and the multi-particle efficiency increase with the scale factor. At $30 \%$ scaling up, the single-particle fake rate increases more than $100 \%$ for both the IBL and the pixel barrel. The multi-particle efficiency saturates once the charge in the cluster is scaled up by $\sim 20 \%$. The saturation occurs as the multi-particle efficiency approaches $100 \%$. When scaling down the charge by $30 \%$, the multi-particle efficiency decreases by more than $20 \%$ in both the IBL and the pixel barrel. The single-particle fake rate also decreases by at least $30 \%$ for both the IBL and the pixel barrel. Effectively by increasing (decreasing) the total cluster charge the cluster will look more (less) like a multi-particle cluster.

The type of variation mostly impacting the resolution of the position NN estimates is the one which affects the threshold of the time-over-threshold (ToT) read-out. This variation is probing the sensitivity to pixel ToT threshold variations. It is performed by subtracting charge values of 0 (default), 100, 250, 500, 750 and 1000 electrons from each pixel in the cluster. If the resulting 


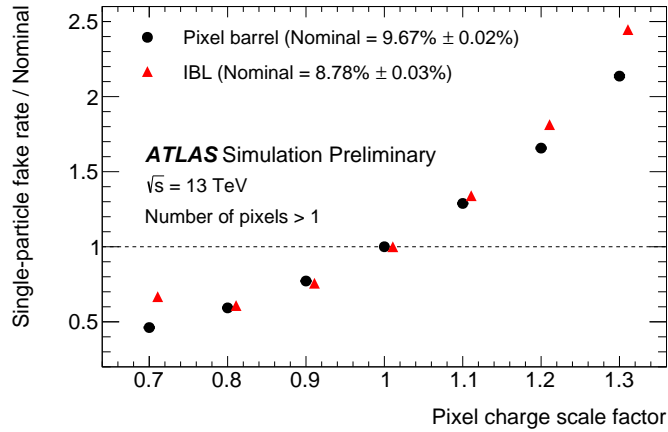

(a) Single-particle cluster, used by multiple tracks

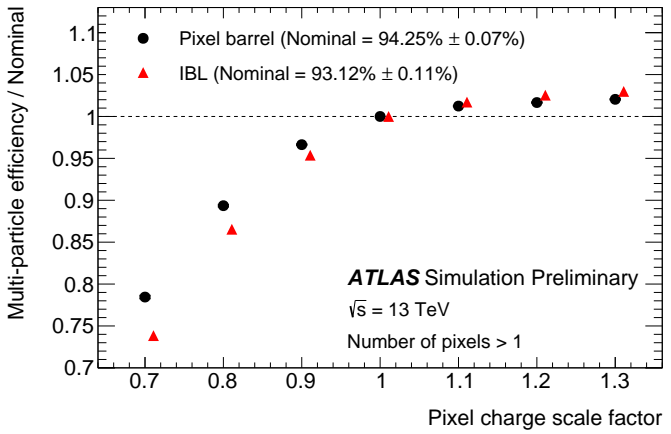

(b) Multi-particle cluster, used by multiple tracks

Figure 1: Performance of the pixel neural network used to identify clusters created by multiple charged particles, as a function of constant coherent scaling of the charge in each pixel in the cluster. The left figure shows the rate at which the neural network wrongly identifies clusters with one generated particle as clusters with multiple particles. The right figure shows the rate at which the neural network correctly identifies clusters generated by multiple particles as such. The points have been staggered along the $x$-axis for visibility. Figure from Ref. [2].

charge is below the ToT threshold (2500 electrons for the IBL, 3500 electrons for the remainder of the pixel barrel) the charge in that pixel is set to zero. The magnitude of the variations is based on the accuracy of the charge calibration scale. In the simulated events, there are a small fraction of clusters where all pixels in the cluster fall below the threshold and subsequently not studied further. The dimensions of the cluster are determined prior to the modification of the cluster, to ensure studying the same subset of clusters before and after the variation.

The single-particle position NN residual distribution widths as a function of the number of electrons removed from each pixel in the cluster is shown in Fig. 2. The resolution degrades with increasing charge subtraction. For the local $x$ direction, the IBL is more sensitive to the variation than the pixel barrel and reaches roughly 30\% degradation at 600 electrons subtraction. The degradation is expected since this variation a) alters the relative weighting between pixel charges in the cluster and $b$ ) removes information when dropping pixels going below threshold. The same behaviour is observed for the two- and three-particle position NNs in both directions, but less pronounced $(<5 \%$ everywhere).

For both directions and for all particle multiplicities, the difference in means between the residual distribution and the corresponding nominal distribution show no or very small dependence $(<3 \mu \mathrm{m})$ under all tested variations.

\section{Summary and Conclusions}

The robustness of the artificial neural networks (NNs) used for identifying clusters with multiple charged particles and assigning positions in the ATLAS pixel detector has been studied. The particle multiplicity NN can be considered robust against variations which do not change the total 


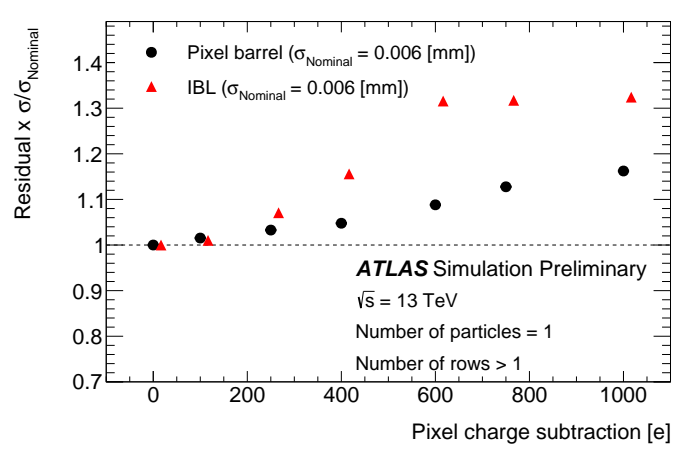

(a) Residual width local $x$

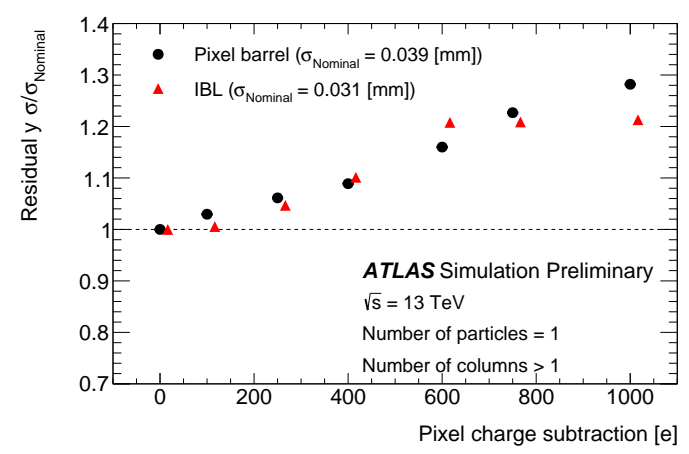

(b) Residual width local $y$

Figure 2: Performance of the pixel neural networks used to estimate positions of particles in a pixel cluster, as a function of a constant subtraction of charge from each pixel in the cluster, with subsequent removal of pixels for which the modified charge goes below threshold. The $y$ axes show the ratio between the width of the residual distribution after the variation and the nominal width, where the residual is calculated as the difference between the $\mathrm{NN}$ estimate and the intersection with the module of the simulated particle. The points have been staggered along the $x$-axis for visibility. "Rows" ("columns") indicates the number of pixels in the cluster in the local $x(y)$ direction. The number of rows and columns are calculated before the variation is performed. Figure from Ref. [2].

charge of the cluster. However, it is sensitive to the uncertainty in the charge calibration scale. Charge mis-calibration has the potential to impact track reconstruction performance. The resolution of the position NN estimates is sensitive to subtracting charge from the cluster, with potential pixel removal if the modified pixel charges go below threshold. For the other variations, the NNs do not reach the same levels of degradation, or do so only for excessive variation magnitudes.

\section{References}

[1] ATLAS Collaboration. Concepts, Design and Implementation of the ATLAS New Tracking (NEWT). http://cdsweb.cern.ch/record/1020106.

[2] ATLAS Collaboration. Robustness of the Artificial Neural Networks Used for Clustering in the ATLAS Pixel Detector. https://cds.cern.ch/record/2104514.

[3] ATLAS Collaboration. The ATLAS Experiment at the CERN Large Hadron Collider. JINST 3 (2008) S08003.

[4] ATLAS Collaboration. ATLAS Insertable B-Layer Technical Design Report. CERN-LHCC-2010-013. ATLAS-TDR-19. http://cdsweb.cern.ch/record/1291633.

[5] ATLAS Collaboration. The Optimization of ATLAS Track Reconstruction in Dense Environments. https://cds.cern.ch/record/2002609.

[6] ATLAS Collaboration. A neural network clustering algorithm for the ATLAS silicon pixel detector. JINST 9 (2014) P09009. [hep-ex/1406.7690]

[7] ATLAS Collaboration. Measurement of performance of the pixel neural network clustering algorithm of the ATLAS experiment at $\sqrt{s}=13 \mathrm{TeV}$. https://cds.cern.ch/record/2054921. 